\section{Fatal retroperitoneal gas gangrene complicating colonoscopic polypectomy without bowel perforation in a healthy adult}



Fig. 1 Abdominal computed tomography (CT) scan images in a previously healthy adult post-polypectomy showing a retropneumoperitoneum, with the emphysema also involving the right psoas muscle.

retropneumoperitoneum, which was also dissecting muscle planes in the right leg and scrotum. The emphysema also extended into the thorax. Staining of the previously removed tissue showed Grampositive bacilli, indicating possible gas gangrene infection. A second laparotomy with extensive retroperitoneal debridement was performed but, despite resuscitation efforts, the patient died 36 hours after admission.

Post-mortem cultures were positive for $C$. perfringens. An autopsy showed massive intra-abdominal bleeding, with destruction and liquefaction of the retroperitoneal muscles. No macroscopic bowel perforation was found. Histological examination of the polyps did not identify a malignancy.

Gas gangrene is a soft-tissue infection caused by Clostridium spp.[4,5]. A defining diagnostic feature is gas dissecting into the muscle bellies, and CT scanning is especially useful to diagnose this in an intra-abdominal location [6]. A literature review identified one other case of retroperitoneal gas gangrene associated with colonoscopic polypectomy [7]. Therefore, although CT scan images showing retroperitoneal gas after a colonoscopy have been associated with colonic perforation $[8,9]$, gas gangrene should also be considered in the differential diagnosis, especially after polypectomy and when the gas involves the muscle belly.

Endoscopy_UCTN_Code_CPL_1AJ_2AC

Competing interests: None

Colonoscopy is a commonly performed procedure that is rarely associated with severe complications, especially not lifethreatening infections [1-3]. We report a fatal outcome caused by Clostridium perfringens after colonoscopic polypectomy in a 58-year-old healthy adult. The colonoscopy was performed as part of a public health screening program for colorectal cancer studying fecal occult blood, and during the procedure four polyps were removed.

The patient was readmitted 12 hours after the colonoscopy complaining of severe back and right-sided abdominal pain. Physical examination was normal except for abdominal tenderness. A computed tomography (CT) scan revealed the presence of extensive retroperitoneal gas, which was also involving the right psoas muscle ( Fig.1). A bowel perforation was suspected, so an urgent laparotomy was indicated. Surgical exploration revealed extensive retroperitoneal emphysema with a parietocolic hematoma, but no evidence of a macroscopic bowel perforation. The hematoma was drained and microbiological samples were collected. Antibiotic treatment with imipenem was started.

In the immediate postoperative period the patient developed hemolysis, and severe respiratory and renal failure. A further CT scan revealed worsening of the

\section{Evelyn Shaw ${ }^{1}$, Raquel Reyes ${ }^{2}$, Antonia Bonet ${ }^{2}$, Lucia Garcia-Huete ${ }^{2}$, Alberto Pasqualetto ${ }^{2}$, Fe Tubau ${ }^{3}$, Jordi Carratalà ${ }^{1}$}

${ }^{1}$ Department of Infectious Diseases, Hospital Universitari de Bellvitge, Barcelona, Spain and Spanish Network for Research in Infectious Diseases (REIPI RD12/0015), Instituto de Salud Carlos III, Madrid, Spain

2 Department of Anesthesia, Hospital Universitari de Bellvitge, Barcelona, Spain

${ }^{3}$ Department of Microbiology, Hospital Universitari de Bellvitge, Barcelona, Spain and CIBERES (CIBER de Enfermedades Respiratorias), Instituto de Salud Carlos III, Madrid, Spain 


\section{References}

1 Fisher DA, Maple JT. ASGE Standards of Practice Committee et al. Complications of colonoscopy. Gastrointest Endosc 2011; 74: $745-752$

2 Warren JL, Klabunde $C N$, Mariotto $A B$ et al. Adverse events after outpatient colonoscopy in the Medicare population. Ann Intern Med 2009; 150: 849-857

3 Nelson DB. Infectious disease complications of GI endoscopy: part I, endogenous infections. Gastrointest Endosc 2003; 57: $546-$ 556

4 Stevens DL, Bisno AL, Chambers HF et al. Practice guidelines for the diagnosis and management of skin and soft-tissue infections. Clin Infect Dis 2005; 41: 1373-1406
5 Stevens DL, Aldape MJ, Bryant AE. Life-threatening Clostridial infections. Pathogenesis and toxins. Anaerobe 2012; 18: 254-259

6 Chapnick EK, Abter EI. Necrotizing soft-tissue infections. Infect Dis Clin North Am 1996; 10: $835-855$

7 Boenicke L, Maier M, Merger M et al. Retroperitoneal gas gangrene after colonoscopic polypectomy without bowel perforation in an otherwise healthy individual: report of a case. Langenbecks Arch Surg 2006; 391: $157-160$

8 Bakker J, van Kersen F, Bellaar Spruyt J. Pneumopericardium and pneumomediastinum after polypectomy. Endoscopy 1991; 23: $46-47$

9 Daniels IR, Sullivan T, Hale J. Retroperitoneal gas after colonoscopy. J R Soc Med 1999; 92: $21-22$
Bibliography

DOI http://dx.doi.org/

10.1055/s-0033-1344990

Endoscopy 2014; 46: E91-E92

(c) Georg Thieme Verlag KC

Stuttgart · New York

ISSN 0013-726X

\section{Corresponding author}

\section{Evelyn Shaw, MD}

Department of Infectious Diseases Hospital Universitari de Bellvitge

Feixa Llarga s/n

L'Hospitalet de Llobregat 08907

Barcelona

Spain

Fax: +34-93-2607274

eshawp@gmail.com 